\title{
SEMINARIO MATEMATICO E FISICO DI MILANO
}

Giuseppe Occhialini (Direttore) - Giovanni Ricci (Direttore) - ERManNo Marchionna (Segretario) - Luigi Amerio - GiUseppe Belardinelli - Italo Bertolini - EmanUele Biondi - ANtonio Bisi Luisa Bisi Zappa - Giuseppe Bolla - Ercole Bottani - Piero Caldirola - Lionello Cantoni - Francesco Carassa - Corrado Casci Duilio Citrini - Emilio Clauser - Laura Colli - Marco Cugiani Mariano Cunietti - Luigi Dadda - Modesto Dedò - Alessandro Dei Poli - Giulio de Marchi - Antongiulio Dornig - Fiorenzo DuiMio - Ugo FACChini - BRuno Finzi - Leo Finzi - EtToRe Fiorini Sergio Gallone - Umberto Gasapina - Emilio Gatti - Guido Gotusso - Giuseppe Grandori - Elisa Grandori Guagenti - Ileana IORI - ENNIO LAZZARINI - PIERo LOCATELli - ANGElo LOINGER - LUCIA Lombardi Tallone - Antonio Lovati - Martina Lovati Panetti Lorenzo Lunelli - Giorgio Malgarini - Carlo Felice manara Cesarina Marchionna Tibiletti - arnaldo Masotti - Giuseppina Masotti Biggiogero - Emilio Massa - Giovanni Melzi - Emilio Montaldi - Giulio Natta - Costanza Occhialini Dilwort - Maria Pastori - Umberto Pellegrini - Giuseppe Peretti - Franca Maria Peretti Graiff - Maria Luisa Pini de Socio - Roberto Piontelli - Giovanni Polvani $(\dagger$ - Livio Porcu - Ermenegildo Preti Gianmaria Prosperi - Giovanni Prouse - Adolfo Quilico - Gaetano Rodriquez - Carlo Salvetti - Livio Scarsi - MrChele SCE OtTorino Sesini - Mario Silvestri - Fulvia Skof - LUigi SolaINI Piergiorgio Sona - Carlo Succi - Guido Tagliaferri - Giorgio TaLENTI - Bartolomeo Todeschini - Paolo Udeschini - Elisa UdeSCHINI BRINIS - CARLo VENINI - FRANCESCO ZAGAR. 